\title{
Backprojection informatics of RU Monocerotis-type binary system light curves
}

\author{
V. Bahýl ${ }^{1}$, M. Kodrík ${ }^{2}$, E. Balážová2 ${ }^{2}$ Pham Van Tinh ${ }^{3}$, \\ O. Vacek ${ }^{2}$ and M. Vargovská ${ }^{2}$ \\ 1 Observatory Júlia, 962 01 Zvolenská Slatina, Slovak Republic (E-mail: \\ basoft@zv.psg.sk) \\ 2 Technická Univerzita vo Zvolene, Masaryka 24, 96001 Zvolen, Slovak \\ Republic \\ 3 Faculty of Information Technology, Nong Lam University, Thu Duc district, \\ Ho Chi Minh City, Vietnam
}

Received: September 27, 2019; Accepted: December 2, 2019

\begin{abstract}
This paper presents the results of our mathematical experiment with light curves of variable stars with large apsidal motion. We have treated our photometric data for different positions of longitude of periastron with our reconstruction algorithms.
\end{abstract}

Key words: informatics - computer tomography - binaries: eclipsing

\section{Introduction and methods}

The mathematical basis of computer tomography $(\mathrm{CT})$ has penetrated many scientific disciplines since the times of J.K. A. Radon, G. Hounsfield and A. MacLeod Cormack. Astronomy is no exception. For many years we have had at our disposal the results of Marsh (2001), Richards et al. (2014) and co-workers. All these authors dealt with applications of reconstruction algorithms in some very interesting branches of astronomy. We began working in this branch of science only recently when we studied the changes in the primary minimum of the $\beta$ Lyrae system, in a similar way to the study of light curves of accretion discs in eclipsing cataclysmic variables (Horne \& Stiening, 1985). At this point we would like to emphasize that our scientific effort is to study the minima of $\beta$ Lyrae in a fully independent way from that discussed by other authors. However, their results fully support our work even though we have only published our results in their full complexity just recently (Bahýl et al., 2019). These first results encouraged us to continue the study of full light curves of selected variables with the CT method.

Plenty of books and papers on the subject of tomographic reconstruction of the internal structures of very different objects have been written. However, in our opinion, the world's best book in this field is that by Kak \& Slaney (1988). In accordance with the formalism given in this book we have realized our own software with the $\mathrm{C}++$ Builder. 
Of course, there is not enough space in this paper to present the formalism used in our work in full detail. However, we would like to stress that we have not worked with a so called "black box" downloaded from the internet! We have written our own software based on relevant mathematical fundamentals.

\section{Data results and conclusions}

There are many types of variable stars, but in our experience, not all of them are suitable for tomographic reconstruction of their light curves. We have selected Algol-type variables with very large apsidal motion. A prototype of such systems is RU Monocerotis, mainly because it has been well observed from the beginning of the last century and its apsidal motion is very large (Martynov, 1966).

The basic task in CT is to get so called projections of the tomographed object, and subsequently, to obtain a reconstruction of the internal structure of the object. In accordance with the general approach, we have accepted the light curves of the selected system at different longitudes of periastron as the projections.

At this point we would like to mention that we have at our disposal many observational data listed on the Czech Astronomical Society web page http://var2. astro.cz.

All relevant data which we have used are referenced on that site.

We have decided to work with 18 projections in the process of back-projecting and internal structure reconstruction. In the process of modelling the given light curve with the BM3 software system we have determined in the longitudes of periastron. An example of our set of light curves for the RU Mon-type system MU Cas is shown in Fig. 1. When we put these data as input into the tomo-
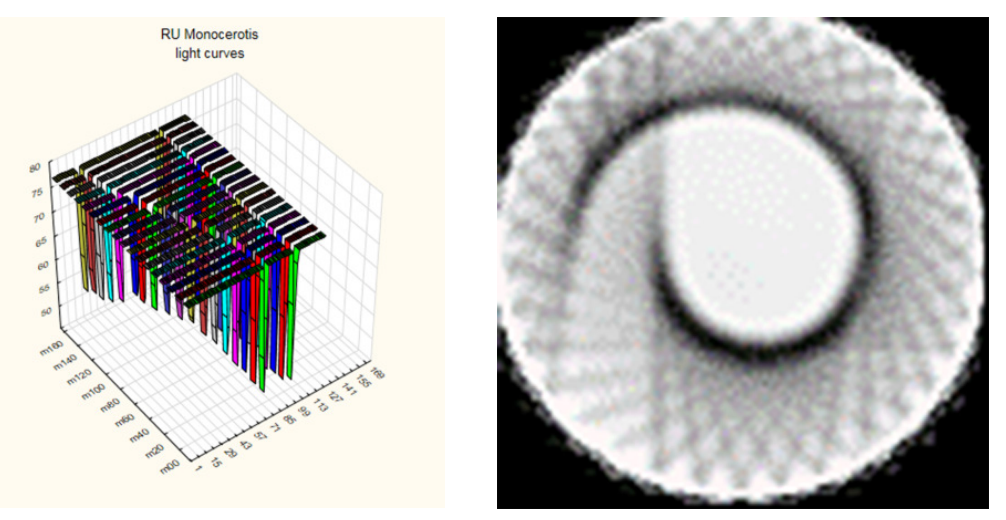

Figure 1. Left: the set of the MU Cas light curves for different longitudes of periastron. Right: tomographic reconstruction of the set of light curves of the MU Cas system. 
graphic reconstruction software, we obtain the result in the right-hand panel. There are two semicircles. The half circle which corresponds to the secondary minima is larger and begins at the right-hand end of the smaller half circle which corresponds to the primary minima. The lines radiating from the semicircles are so called ghosts which appear because we only use eighteen projections. These ghosts can be easily suppressed computationally.

We have also reconstructed the system V456 Ophiuchi. This system has zero eccentricity, and as expected, in accordance with the theory, both half circles establish a single full circle.

Since its inception CT has found its way into almost all branches of human activity, including astronomy. We have successfully used Doppler tomography in astronomy. We feel that our effort demonstrates that we have the possibility of applying computer tomography to the analysis of light curves of at least some special kinds of variable stars. Or better still, we hope that CT light curve analyses can yield new insights into the problems of changes of the positions of light curve secondary minima.

Lastly we would like to emphasize that we are very familiar with the WilsonDevinney, Phoebe and MP3 methodologies to determine close binary system parameters. With our work we are not trying to change, improve or replace these methods. Simply, our goal is to take a very small additional step and apply the CT method to obtain system parameters. In this we feel we have been successful.

Acknowledgements. We thank to Dr. M. Husárik for valuable advice, help and assistance with the preparation of this article in the $\mathrm{AT}_{\mathrm{E}} \mathrm{X}$ environment.

\section{References}

Bahýl, V., Gajtanská, M., Hanisko, P., \& Van, T. P., The Informatics in the Doppler Tomography Modeling of the Envelopes of the Close Binaries Systems. 2019, Open European Journal on Variable Stars, 197, 37

Horne, K. \& Stiening, R. F., Eclipse maps of the accretion disc in RW Trianguli. 1985, Monthly Notices of the Royal Astronomical Society, 216, 933

Kak, A. C. \& Slaney, M., Principles of computerized tomographic imaging IEEE Press. 1988, New York

Marsh, T. R., Doppler tomography. 2001, in Astrotomography (Springer), 1-26

Martynov, D. Y., RU Monocerotis. Further Data on the Motion of the Line of Apsides. 1966, Soviet Astronomy, 9, 939

Richards, M. T., Cocking, A. S., Fisher, J. G., \& Conover, M. J., Images of gravitational and magnetic phenomena derived from two-dimensional back-projection Doppler tomography of interacting binary stars. 2014, The Astrophysical Journal, 795, 160 